\title{
Clinicopathological Characteristics of Breast Ductal Carcinoma In Situ: An Analysis of Chinese Population of 617 Patients
}

\author{
Guangmin Mao, ${ }^{1,2,3}$ Xiu-hua Shi, ${ }^{1,2,4}$ Xiaofang Wang, ${ }^{1,2}$ Xiaomeng Zhang, ${ }^{1,2}$ \\ Xingxing Chen, ${ }^{1,2}$ Jinli Ma $\mathbb{D}^{1,2}$ Xiaoli Yu, ${ }^{1,2}$ Zhen Zhang, ${ }^{1,2}$ and Xiaomao Guo ${ }^{1,2}$ \\ ${ }^{1}$ Department of Radiation Oncology, Fudan University Shanghai Cancer Center, Fudan University, Shanghai 200032, China \\ ${ }^{2}$ Department of Oncology, Shanghai Medical College, Fudan University, Shanghai, China \\ ${ }^{3}$ Department of Radiation Oncology, Longhua Hospital Shanghai University of Traditional Chinese Medicine, \\ Shanghai 200032, China \\ ${ }^{4}$ Department of Radiation Oncology, The No. 2 People's Hospital of Wuhu City, Wuhu, Anhui Province, China
}

Correspondence should be addressed to Jinli Ma; jinli.ma@aliyun.com

Received 14 August 2020; Revised 4 December 2020; Accepted 25 December 2020; Published 5 January 2021

Academic Editor: Raffaele Palmirotta

Copyright (c) 2021 Guangmin Mao et al. This is an open access article distributed under the Creative Commons Attribution License, which permits unrestricted use, distribution, and reproduction in any medium, provided the original work is properly cited.

\begin{abstract}
Background. The purpose of this study was to describe the clinicopathological characteristics of breast DCIS in Chinese women and compare with that of patients in western countries. Method. From December 2005 to December 2015, 617 women diagnosed with pure DCIS after surgery at our institution were enrolled, and the clinicopathological characteristics were described. Results. In this study, the percentage of patients detected on screening, diagnosed at $\leq 50$ years of age, with tumor size $\leq 2.0 \mathrm{~cm}$, and with lowintermediate grade was $39.4 \%, 56.7 \%, 72.6 \%$, and $77.4 \%$, respectively, as compared to $50-80 \%, 20-30 \%, 70-90 \%$, and $40-60 \%$ in published reports from western countries. The percentage of ER-positive patients was $76.3 \%$ in this study, which is similar to the mean expression rate of ER (mean: 68.7\%, range: 49-96.6\%) reported previously. Conclusions. The clinicopathological characteristics of Chinese DCIS patients include less detection on screening, younger age at diagnosis, and more low-intermediate nuclear grade.
\end{abstract}

\section{Introduction}

With the introduction of mammography screening, the incidence of ductal carcinoma in situ (DCIS) of breast has increased dramatically in last decades and now comprises $20-25 \%$ of all detected breast cancers [1,2]. Although it is uncertain what exact rate of DCIS will progress to invasive cancer, $14-53 \%$ of the DCIS patients who were misdiagnosed as benign lesions turned out to be invasive breast cancer 10 years later [3]. Clinicopathological factors, such as age, estrogen receptor (ER) and/or progesterone receptor (PR) status, nuclear grade, and tumor size, were believed to be associated with risk of local recurrence (LR) and progression to invasive cancer in DCIS patients $[4,5]$. It was reported that clinicopathological characteristics of invasive breast cancer patients in China differ from that of patients from western countries [6], which reminded us that the characteristics of Chinese DCIS patients may be unique as well. No large-scale population-based study on the clinicopathological characteristics of Chinese DCIS patients has been reported previously.

The purpose of this study was to describe the clinicopathological characteristics of breast DCIS in Chinese women and compare with that of patients in western countries.

\section{Methods}

2.1. Study Population. Of all the breast cancer patients diagnosed at our institute from December 2005 to December 2015, 1392 patients were identified to have breast DCIS after surgery. Patients who were male $(n=1)$, or with DCIS with microinvasion $(n=271)$, or diagnosed with invasive breast cancer after further surgery $(n=417)$ were excluded. Only primary pure DCIS patients were included in our study. All the primary pure DCIS diagnosis and pathological 
characteristics were confirmed by at least two pathologists based on surgical specimens. We also excluded 86 patients either for the history of previous invasive breast cancer or lack of information. The final cohort included 617 patients.

2.2. Clinicopathological Characteristics. Clinical characteristics, such as the detection method and age at initial diagnosis, were collected. The detection method referred to screening-detected by imaging (e.g., ultrasonography and mammography) and clinical symptoms (e.g., a noticed lump, nipple discharge, and breast pain).

Pathological characteristics including the tumor size, nuclear grade, ER status, PR status, and surgical margins were extracted from the original pathological reports based on surgical specimens. The tumor size was defined as the largest histologic dimension, including any discontinuous areas (e.g., multifocal lesions). The nuclear grade was pathologically assessed and classified as "low," "intermediate," and "high." Low/intermediate grade was defined by the presence of nuclear grades 1 or 2 with limited or no foci of necrosis. High grade was defined by the presence of nuclear grade 3 atypia and comedo-type necrosis that was zonal (i.e., present in contiguous ductal spaces).

ER status and PR status were evaluated by immunohistochemistry (IHC) staining. A $1 \%$ cutoff value was used to dichotomise cases into positive and negative [7]. The surgical margin was pathologically assessed and classified as "negative" with margin width $\geq 2 \mathrm{~mm}$, "close" with margin width between 0 and $2 \mathrm{~mm}$, and positive.

2.3. Definition of Patient Subgroups. Patient subgroups were defined according to the detection method, age at diagnosis, tumor size, nuclear grade, and hormone receptor status.

Patients detected by imaging at time of screening or incidentally found in tissue of an otherwise benign biopsy were classified as the "screening" group, and patients who presented with clinical signs at diagnosis were classified as the "symptomatic" group. A cutoff value of 50 years for age at initial diagnosis was used to define age subgroups $(\leq 50$ vs $>50$ ), and a cutoff value of $2.0 \mathrm{~cm}$ was used to define subgroups for tumor size $(\leq 2.0 \mathrm{~cm}$ vs $>2.0 \mathrm{~cm})$. Patients with lowgrade, low-intermediate grade, or intermediate grade DCIS were categorized as the "low-intermediate grade" group, whereas patients with intermediate-high grade or high-grade DCIS were categorized as the "high-grade" group.

\section{Statistical Analysis}

Descriptive statistics were used to analyze the clinicopathological characteristics of Chinese DCIS patients. All statistical analyses involved in this study were performed using SPSS Version 22.0 (IBM Corporation, Armonk, NY, USA).

\section{Results}

The study population consisted of 617 women who presented with pure DCIS and was treated at our institute. Of them, 470 patients $(76.2 \%)$ underwent total mastectomy, and the rest underwent breast-conserving surgery (BCS) with $(n=106)$ or without $(n=41)$ adjuvant radiotherapy. Following local therapy, 328 patients $(53.2 \%)$ received adjuvant endocrine therapy. Patients' clinicopathological characteristics are listed in Table 1.

Of all the patients, $243(39.4 \%)$ were categorized in the "screening" group, and 362 (58.7\%) were categorized in the "symptomatic" group. The median age at the time of initial diagnosis was 48 years (range: 19-88 years). Using 50 years of age at initial diagnosis as a cutoff value, 350 patients (56.7\%) were classified as the " $\leq 50$ years" group, and 267 patients $(43.3 \%)$ were classified as the " $>50$ years" group. 606 patients (98.2\%) had unilateral disease, and 601 patients (97.4\%) presented with unicentric lesion. The tumor size was measuring $\leq 2.0 \mathrm{~cm}$ in 448 cases $(72.6 \%), 2.1-5.0 \mathrm{~cm}$ in 120 patients $(19.4 \%)$, and $>5 \mathrm{~cm}$ in 6 patients $(9.7 \%)$. The nuclear grade was "low-intermediate" in 463 patients (75.0\%) and "high" in 135 patients (21.9\%). On IHC staining, ER was positive in 471 patients (76.3\%) and PR positive in 426 patients (69.0\%).

Table 2 shows comparison of clinicopathological characteristics of patients with pure DCIS between this and previous studies.

Table 2 compared our data with published data from western countries by the year of 2015 [8-17]. In brief, the comparison showed that the percentage of patients detected on screening was only $39.4 \%$ in our study, as compared to $50 \%$ to $80 \%$ in previous studies; the percentage of patients with age $\leq 50$ years in our study was around $20 \%$ higher than that reported in other studies. The percentages of patients with tumor size $\leq 2.0 \mathrm{~cm}$ were $78.1 \%$, which is similar to findings from SEER study, but is $10 \%$ lower than that reported by other studies including NSABP B-24 and EORTC. The percentage of patients with low-intermediate grade disease was $77.4 \%$ in this study, which is around $10 \%-40 \%$ higher than that reported in other studies. Great variations were observed to exist in the rates of ER and PR expression among studies.

\section{Discussion}

Previous data demonstrated that striking differences existed in the incidence rates and clinicopathological features of invasive breast cancer among women from diverse countries and different ethnic and genetic backgrounds [6]. However, it is not clear whether a similar situation exists for noninvasive breast cancer. The current study analyzed the clinicopathological characteristics of 617 Chinese women with pure DCIS, in comparison with published data of patients from western countries. To our knowledge, this is so far the first population-based study dealing with the differences in clinicopathological features of noninvasive breast cancer among women from China and western countries.

5.1. Detection Method. A significant negative association was reported to exist between screening-detected DCIS and subsequent invasive interval cancers, and the detection and treatment of DCIS was considered to be worthwhile in prevention of future invasive disease [18]. The DCIS with 
TABle 1: Clinicopathological characteristics of Chinese DCIS patients $(n=617)$.

\begin{tabular}{|c|c|c|}
\hline Characteristics & No. of cases & $\%$ \\
\hline \multicolumn{3}{|l|}{ Detection method } \\
\hline Symptomatic & 362 & 58.7 \\
\hline Screening & 243 & 39.4 \\
\hline NA & 12 & 1.9 \\
\hline \multicolumn{3}{|l|}{ Age (years) } \\
\hline Median & 48 & \\
\hline$\leq 40$ & 122 & 19.8 \\
\hline $41-50$ & 228 & 37.0 \\
\hline $51-60$ & 151 & 24.5 \\
\hline $61-70$ & 81 & 13.1 \\
\hline$>70$ & 35 & 5.7 \\
\hline \multicolumn{3}{|l|}{ Tumor size $(\mathrm{cm})$} \\
\hline$\leq 1.0$ & 213 & 34.5 \\
\hline $1.1-2.0$ & 235 & 38.1 \\
\hline $2.1-5.0$ & 120 & 19.4 \\
\hline$>5.0$ & 6 & 9.7 \\
\hline NA & 43 & 7.0 \\
\hline \multicolumn{3}{|l|}{ Nuclear grade } \\
\hline Low-intermediate & 463 & 75.0 \\
\hline High & 135 & 21.9 \\
\hline NA & 19 & 3.1 \\
\hline \multicolumn{3}{|l|}{ ER status } \\
\hline Positive & 471 & 76.3 \\
\hline Negative & 119 & 19.3 \\
\hline NA & 27 & 4.4 \\
\hline \multicolumn{3}{|l|}{ PR status } \\
\hline Positive & 426 & 69.0 \\
\hline Negative & 168 & 27.2 \\
\hline $\mathrm{NA}$ & 23 & 3.7 \\
\hline
\end{tabular}

DCIS: ductal carcinoma in situ; NA: not available; ER: estrogen receptor; PR: progesterone receptor; IHC: immunohistochemistry.

clinical symptoms was believed to be biologically different from screening-detected DCIS, and the clinical symptoms might be a reaction to "early" invasive breast cancer in tumor microenvironment [5]. The detection method was related to the LR rate in pure DCIS cases.

The percentage of screening-detected patients in our study was much lower than that in published data. Factors that were associated with lower rates of screening might include not being covered by medical insurance, lack of screening awareness, fear of mammography X-ray, and inadequate system level interventions. To enhance the screening rates, efforts should be made to promote breast health education, publicize the breast cancer screening guideline, and increase access to breast cancer screening services among low-income populations by enrolling in lowcost or free mammography programs.

5.2. Age at Diagnosis. The age at diagnosis was proven to be an independent prognostic factor for LR in patients with pure DCIS after BCS and was combined with other factors by Van Nuys Prognostic Index (VNPI) to predict for risk of LR [19]. Young age, especially $\leq 40-50$ years, was associated with a higher risk of LR. The percentage of patients with age $\leq 50 \mathrm{y}$ in our study was around $20 \%$ higher than that reported in previous studies. It is unknown whether the difference in the percentage of patients aged $\leq 50 \mathrm{y}$ was associated with a difference in the LR rate between China and western countries. The outcome of ongoing follow-up of this cohort of patients will help to answer this question.

5.3. Tumor Size and Nuclear Grade. The tumor size and nuclear grade were reported to be independent prognostic factors that predicted risk of LR among patients with pure DCIS $[20,21]$. Generally, tumor size $\leq 1.5-2.5 \mathrm{~cm}$ and lowintermediate grade represents lower risk of LR [22]. The percentage of patients with tumor size $\leq 2.0 \mathrm{~cm}$ was $72.6 \%$ in this study, which is similar to findings from SEER study [9] but is $10 \%$ lower than that reported by other studies including NSABP B-24 [16, 17] and EORTC [15]. The percentage of patients with low-intermediate grade was $77.4 \%$, which is significantly higher than that reported in other studies listed in Table 2.

The RTOG 9804 randomized clinical trial included women with screen-detected DCIS, $\leq 2.5 \mathrm{~cm}$ size, low to intermediate nuclear grade, resected with margins negative at $\geq 3 \mathrm{~mm}$ and observed an IBTR risk of $6.7 \%$ in the observation arm, compared to $0.9 \%$ in the whole breast irradiation (WBI) arm at a median follow-up of 7.2 years [23]. Similar results were noted in the initial publication of ECOG 5194 trial among patients meeting similar criteria [24]. These inclusion criteria therefore define a group of patients with low-risk DCIS for whom observation confers a low absolute risk of IBTR and for whom the addition of WBI confers a small but measurable absolute benefit in prevention of IBTR.

5.4. Status of Hormone Receptors. The status of hormone receptors (HRs) including ER and/or PR was an independent predictor of the LR risk as well as effect of endocrine therapy. In this study, $76.3 \%$ of patients were ER positive and $69.0 \%$ were PR positive, which is comparable to the mean rate of ER expression (mean: 68.7\%, range: $49-96.6 \%$ ), as reported in a systemic review by Lari and Kuerer from the University of Texas MD Anderson Cancer Center [25]. Similar finding was noted for PR expression. Generally, following local therapy for pure DCIS, 5 years of endocrine therapy with either tamoxifen or an aromatase inhibitor is recommended for ER- and/or PR-positive patients to reduce the risk of ipsilateral breast events and contralateral breast cancer.

5.5. Adjuvant Radiotherapy and Endocrine Therapy. Following BCS, whole breast irradiation (WBI) with or without subsequent boost to tumor bed was reported to reduce $12.0 \%$ absolute risk and $37.5 \%$ relative risk in ipsilateral breast events at 20 years [26]. In this study, the percentage of patients receiving BCS with or without adjuvant radiotherapy was relatively low, as compared to 1991-2010 SEER data. Efforts should be done to promote the shift of treatment patterns from mastectomy to BCS, as well as the shift from WBI to accelerated partial breast irradiation (APBI) for low-risk DCIS patients who meet 


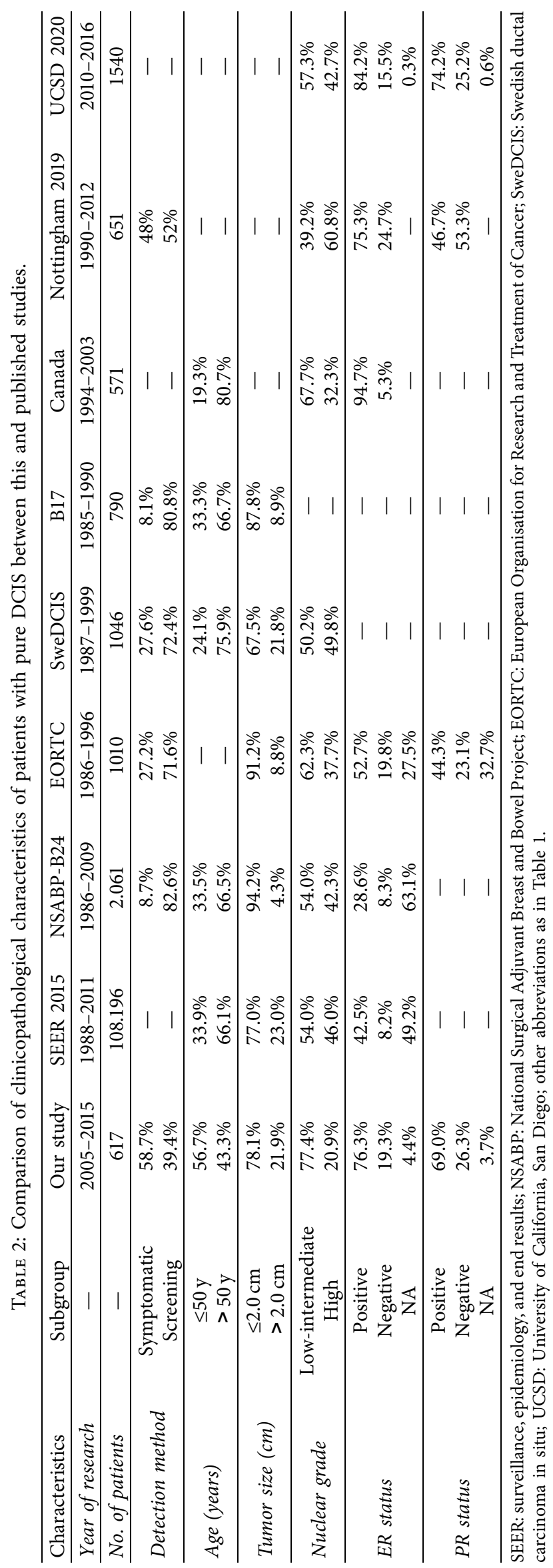


abovementioned criteria, according to 2016 ASTRO guideline [27].

Although ER- and/or PR-positive DCIS patients are mandated to receive endocrine therapy by NCCN guidelines [28], only $69.6 \%$ of HR-positive patients received tamoxifen, toremifene, or aromatase inhibitor in this study. This finding is similar to reports from western countries.

\section{Conclusions}

Compared to published data from western countries, the clinicopathological characteristics of Chinese DCIS patients include the following: (1) lower percentage of patients detected on screening, (2) higher percentage of patients under age of 50 years at diagnosis, and (3) higher percentage of patients with low-intermediate nuclear grade DCIS. Further analysis is warranted to be done to determine whether Chinese DCIS patients have a better prognosis than the patients from western countries.

\section{Data Availability}

The data used to support the findings of this study are available from the corresponding author upon request.

\section{Conflicts of Interest}

The authors declare that they have no conflicts of interest.

\section{Authors' Contributions}

Guangmin Mao, Xiu-hua Shi, and Xiaofang Wang contributed equally to this article.

\section{References}

[1] H. J. Moon, E.-K. Kim, M. J. Kim, J. H. Yoon, and V. Y. Park, "Comparison of clinical and pathologic characteristics of ductal carcinoma in situ detected on mammography versus ultrasound only in asymptomatic patients," Ultrasound in Medicine \& Biology, vol. 45, no. 1, pp. 68-77, 2019.

[2] E. M. Ward, C. E. DeSantis, C. C. Lin et al., "Cancer statistics: breast cancer in situ," CA: A Cancer Journal for Clinicians, vol. 65, no. 6, pp. 481-495, 2015.

[3] B. Erbas, E. Provenzano, J. Armes, and D. Gertig, "The natural history of ductal carcinoma in situ of the breast: a review," Breast Cancer Research and Treatment, vol. 97, no. 2, pp. 135-144, 2006.

[4] S. C. Lester, J. L. Connolly, and M. B. Amin, "College of American pathologists protocol for the reporting of ductal carcinoma in situ," Archives of Pathology \& Laboratory Medicine, vol. 133, no. 1, pp. 13-14, 2009.

[5] S. S. Badve and Y. Gökmen-Polar, "Ductal carcinoma in situ of breast: update 2019," Pathology, vol. 51, no. 6, pp. 563-569, 2019.

[6] S. P. L. Leong, Z.-Z. Shen, T.-J. Liu et al., "Is breast cancer the same disease in Asian and Western countries?," World Journal of Surgery, vol. 34, no. 10, pp. 2308-2324, 2010.

[7] M. E. Hammond, D. F. Hayes, M. Dowsett et al., "American Society of Clinical Oncology/College of American Pathologists guideline recommendations for immunohistochemical testing of estrogen and progesterone receptors in breast cancer," Journal of Clinical Oncology: Official Journal of the American Society of Clinical Oncology, vol. 28, no. 16, pp. 2784-2795, 2010.

[8] S. A. Narod, J. Iqbal, V. Giannakeas, V. Sopik, and P. Sun, "Breast cancer mortality after a diagnosis of ductal carcinoma in situ," JAMA Oncology, vol. 1, no. 7, pp. 888-896, 2015.

[9] L. Holmberg, H. Garmo, B. Granstrand et al., "Absolute risk reductions for local recurrence after postoperative radiotherapy after sector resection for ductal carcinoma in situ of the breast," Journal of Clinical Oncology, vol. 26, no. 8, pp. 1247-1252, 2008.

[10] B. Fisher, J. Costantino, C. Redmond et al., "Lumpectomy compared with lumpectomy and radiation therapy for the treatment of intraductal breast cancer," New England Journal of Medicine, vol. 328, no. 22, pp. 1581-1586, 1993.

[11] E. Rakovitch, S. Nofech-Mozes, W. Hanna et al., "A population-based validation study of the DCIS score predicting recurrence risk in individuals treated by breast-conserving surgery alone," Breast Cancer Research and Treatment, vol. 152, no. 2, pp. 389-398, 2015.

[12] I. M. Miligy, M. S. Toss, K. L. Gorringe et al., "The clinical and biological significance of HER2 over-expression in breast ductal carcinoma in situ: a large study from a single institution," British Journal of Cancer, vol. 120, no. 11, pp. 1075-1082, 2019.

[13] T. J. O'Keefe, S. L. Blair, A. Hosseini, O. Harismendy, and A. M. Wallace, "HER2-overexpressing ductal carcinoma in situ associated with increased risk of ipsilateral invasive recurrence, receptor discordance with recurrence," Cancer Prevention Research, vol. 13, no. 9, pp. 761-772, 2020.

[14] H. Bartelink, J.-C. Horiot, P. Poortmans et al., "Recurrence rates after treatment of breast cancer with standard radiotherapy with or without additional radiation," New England Journal of Medicine, vol. 345, no. 19, pp. 1378-1387, 2001.

[15] N. Bijker, P. Meijnen, J. L. Peterse et al., "Breast-conserving treatment with or without radiotherapy in ductal carcinomain-situ: ten-year results of European organisation for research and treatment of cancer randomized phase III trial 10853-a study by the EORTC breast cancer cooperative group and EORTC radiotherapy group," Journal of Clinical Oncology, vol. 24, no. 21, pp. 3381-3387, 2006.

[16] B. Fisher, J. Dignam, N. Wolmark et al., "Tamoxifen in treatment of intraductal breast cancer: national surgical adjuvant breast and bowel project B-24 randomised controlled trial," The Lancet, vol. 353, no. 9169, pp. 1993-2000, 1999.

[17] A. C. Lo, P. T. Truong, E. S. Wai et al., "Population-based analysis of the impact and generalizability of the NSABP-B24 study on endocrine therapy for patients with ductal carcinoma in situ of the breast," Annals of Oncology, vol. 26, no. 9, pp. 1898-1903, 2015.

[18] S. W. Duffy, A. Dibden, D. Michalopoulos et al., "Screen detection of ductal carcinoma in situ and subsequent incidence of invasive interval breast cancers: a retrospective population-based study," The Lancet Oncology, vol. 17, no. 1, pp. 109-114, 2016.

[19] M. J. Silverstein, "The University of Southern California/Van Nuys prognostic index for ductal carcinoma in situ of the breast," The American Journal of Surgery, vol. 186, no. 4, pp. 337-343, 2003.

[20] I. L. Wapnir, J. J. Dignam, B. Fisher et al., "Long-term outcomes of invasive ipsilateral breast tumor recurrences after lumpectomy in NSABP B-17 and B-24 randomized clinical trials for DCIS," JNCI Journal of the National Cancer Institute, vol. 103, no. 6, pp. 478-488, 2011. 
[21] M. J. Silverstein, A. Barth, D. N. Poller et al., “Ten-year results comparing mastectomy to excision and radiation therapy for ductal carcinoma in situ of the breast," European Journal of Cancer, vol. 31, no. 9, pp. 1425-1427, 1995.

[22] J. Cuzick, I. Sestak, S. E. Pinder et al., "Effect of tamoxifen and radiotherapy in women with locally excised ductal carcinoma in situ: long-term results from the UK/ANZ DCIS trial," The Lancet Oncology, vol. 12, no. 1, pp. 21-29, 2011.

[23] B. McCormick, K. Winter, C. Hudis et al., "RTOG 9804: a prospective randomized trial for good-risk ductal carcinoma in situ comparing radiotherapy with observation," Journal of Clinical Oncology, vol. 33, no. 7, pp. 709-715, 2015.

[24] L. J. Solin, R. Gray, L. L. Hughes et al., "Surgical excision without radiation for ductal carcinoma in situ of the breast: 12-year results from the ECOG-ACRIN E5194 study," Journal of Clinical Oncology, vol. 33, no. 33, pp. 3938-3944, 2015.

[25] S. A. Lari and H. M. Kuerer, "Biological markers in DCIS and risk of breast recurrence: a systematic review," Journal of Cancer, vol. 2, pp. 232-261, 2011.

[26] F. Wärnberg, H. Garmo, S. Emdin et al., "Effect of radiotherapy after breast-conserving surgery for ductal carcinoma in situ: 20 years follow-up in the randomized SweDCIS Trial," Journal of Clinical Oncology, vol. 32, no. 32, pp. 3613-3618, 2014.

[27] C. Correa, E. E. Harris, M. C. Leonardi et al., "Accelerated partial breast irradiation: executive summary for the update of an ASTRO evidence-based consensus statement," Practical Radiation Oncology, vol. 7, no. 2, pp. 73-79, 2017.

[28] S. K. Mardekian, A. Bombonati, and J. P. Palazzo, "Ductal carcinoma in situ of the breast: the importance of morphologic and molecular interactions," Human Pathology, vol. 49, pp. 114-123, 2016. 